\title{
Chinese Neurosurgical Journal reviewer acknowledgement 2015
}

Jizong Zhao

\section{Contributing reviewers}

The editors of Chinese Neurosurgical Journal would like to thank all of our reviewers who have contributed to the journal in volume 1 (2015).

\begin{tabular}{lll} 
Miguel Arraez & Tao Hong & Qing Lan \\
Spain & China & China \\
Shengde Bao & Guangfu Huang & Ting Lei \\
China & China & China \\
Yong Cao & Judy Huang & Gang Li \\
China & United States of America & China \\
Liang Chen & Qinghai Huang & Meihua Li \\
China & China & China \\
Xiaolei Chen & Nan Ji & Xuejun Li \\
China & China & China \\
Gao Chen & Wenqing Jia & Shiting Li \\
China & China & China \\
Hongwei Cheng & Fengzeng Jian & Hai Liu \\
China & China & China \\
Xingli Deng & Yugang Jiang & Jianmin Liu \\
China & China & China \\
Changwu Dou & Chuanlu Jiang & Xiaoqian Liu \\
China & China & China \\
Weiming Duan & Rongcai Jiang & Yunhui Liu \\
United States of America & China & China \\
Hua Feng & Tao Jiang & Xianzhi Liu \\
China & China & China \\
Lei Feng & Jiyao Jiang & Christopher M Loftus \\
United States of America & China & United States of America \\
Guodong Gao & Dezhi Kang & Jun Ma \\
China & China & China \\
Xiaodi Han & Yoko Kato & Chiyuan Ma \\
China & Japan & China \\
\hline
\end{tabular}

Correspondence: zhaojz205@126.com

Department of Neurosurgery, Beijing Tiantan Hospital, Capital Medical

University, Beijing, People's Republic of China 
Jie Ma

China

Basant K Misra

India

Anil Nanda

United States of America

Wai Poon

Hong Kong

Songtao Qi

China

Jianqiang Qu

China

Yan Qu

China

Xiaochuan Sun

China

Bomin Sun

China

Peng Sun

China

Tao Sun

China

Akira Teramoto

Japan

Hengli Tian

China

Yongji Tian

China

Shuo Wang

China

Daming Wang

China

Maode Wang

China
Handong Wang

China

Zhong Wang

China

Renzhi Wang

China

Yunjie Wang

China

Zhenyu Wang

China

Guihuai Wang

China

Junji Wei

China

Qun Wu

China

Zhebao Wu

China

Anding Xu

China

Yulun Xu

China

Ruxiang Xu

China

Hui Yang

China

Xiaofeng Yang

China

Tao Yu

China

Xinguang $\mathbf{Y u}$

China

Shuqing Yu

China
Shengquan Zhan

China

Hongqi Zhang

China

Jianning Zhang

China

Nu Zhang

China

Zhiwen Zhang

China

Jianmin Zhang

China

Yazhuo Zhang

China

Shiguang Zhao

China

Peng Zhao

China

Weiguo Zhao

China

Hongyang Zhao

China

Jiannong Zhao

China

Chunlong Zhong

China

Liangfu Zhou

China

Qichuan Zhuge

China 